\title{
Unlinkability of Sanitizable Signatures
}

\author{
Christina Brzuska, Marc Fischlin, Anja Lehmann, and Dominique Schröder \\ Darmstadt University of Technology, Germany \\ www.minicrypt.de
}

\begin{abstract}
Sanitizable signatures allow a designated party, called the sanitizer, to modify parts of signed data such that the immutable parts can still be verified with respect to the original signer. Ateniese et al. (ESORICS 2005) discuss five security properties for such signature schemes: unforgeability, immutability, privacy, transparency and accountability. These notions have been formalized in a recent work by Brzuska et al. (PKC 2009), discussing also the relationships among the security notions. In addition, they prove a modification of the scheme of Ateniese et al. to be secure according to these notions.

Here we discuss that a sixth property of sanitizable signature schemes may be desirable: unlinkability. Basically, this property prevents that one can link sanitized message-signature pairs of the same document, thus allowing to deduce combined information about the original document. We show that this notion implies privacy, the inability to recover the original data of sanitized parts, but is not implied by any of the other five notions. We also discuss a scheme based on group signatures meeting all six security properties.
\end{abstract}

\section{Introduction}

For a regular signature scheme any modification of the message makes the signature for the modified message invalid. In some applications, though, it may be preferable to support message modifications such that one can still verify the authenticity of the immutable message part, and that only authorized parties can make such changes. Signature schemes having this property are called sanitizable, as introduced by Ateniese et al. [1]. Related concepts have been discussed concurrently in 242320 .

Ateniese et al. 1 discuss the applicability of sanitizable signatures to anonymization of medical data, replacing commercials in authenticated media streams or updates of reliable routing information. They identified five desirable security properties for sanitizable signature schemes. Informally, these are:

UnForgeability. Says that no one except for the honest signer and sanitizer can create valid signatures.

ImmutABiLity. Demands that even a malicious sanitizer cannot change message parts which have not been marked as modifiable by the signer.

PrIVACY. Prevents an outsider to recover the original data of sanitized message parts. 
Transparency. Covers the indistinguishability of signatures created by the signer or the sanitizer.

Accountability. Refers to the inability of a malicious signer or sanitizer to deny authorship.

Brzuska et al. 3] define these five properties with game-based approaches formally and relate them, showing that accountability implies unforgeability and transparency implies privacy; all other properties are independent. They also prove a modification of the scheme by Ateniese et al. [1] to be secure according to these five properties.

Unlinkability. Here we discuss that an additional property may be useful in some settings. We call this property unlinkability and motivate it by the following example (see also Figure 1): Assume that we have signed medical records and at some point we anonymize the data by redacting the personal information of the patients like names, addresses etc. At some other time, say for revenues reasons, we remove the actual medical treatments and leave only the personal information. Then one should not be able to link these data through the (sanitized) signatures and therefore reconstruct the full records. However, previous schemes like the one by Brzuska et al. 3] and, for example, the ones in 211110] in fact allow such attacks. They are usually based on chameleon hashes which remain unchanged for the sanitization step and thus allow to identify two sanitized signatures derived from the same signature through the hash value. Other constructions like the one in 23 . even come with an explicit document identifier, allowing to link sanitized messages easily.

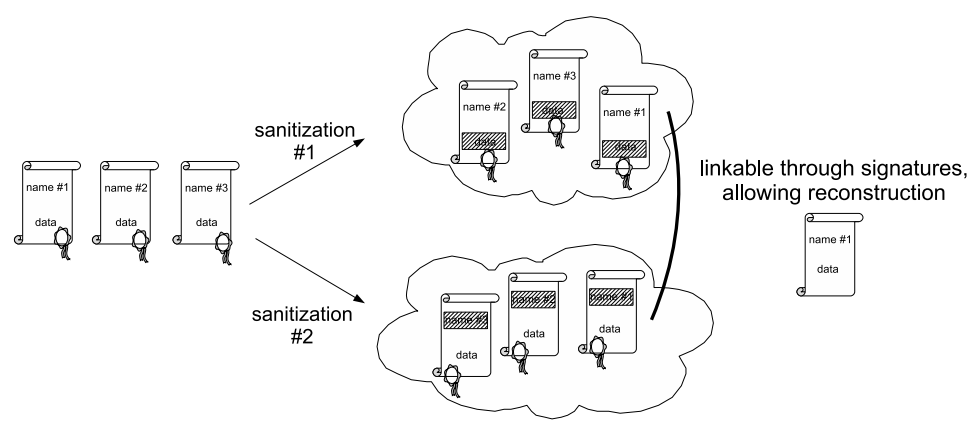

Fig. 1. Linkability problem

We hence introduce a formal definition of unlinkability and relate it to the previously given notions. It turns out that unlinkability is not implied by any of the other properties, but vice versa implies privacy. The reason is that privacy prevents an adversary of recovering the original data for sanitized parts, and violation of this property also enables the adversary to reconstruct and to link messages easily. 
Construction. We then present a construction of a sanitizable signature scheme obeying all six properties, including unlinkability. The idea is fundamentally different from previous approaches which usually rely on chameleon hashes. In our case the signer first signs the fixed parts with a regular signature scheme. For the modifiable parts the signer and the sanitizer use a group signature scheme 13], i.e., a signature scheme which allows to sign anonymously on behalf of the group but such that a group manager can revoke the identity of the user that has signed [5]. In our case the group only consists of the signer and sanitizer, and the signer also incarnates the group manager. If the sanitizer later changes (some of) the modifiable message parts it can create a new group signature and replace the signer's group signature.

The anonymity of the group signature scheme in our context guarantees transparency (the indistinguishability of signatures originating from the signer and the sanitizer). The possibility to identify a group member by the group manager (i.e., the signer in our case) supports sanitizer-accountability, i.e., the ability to provide a proof that the sanitizer has created the signature. Signer-accountability is provided by the non-frameability of the group signature scheme which prevents a malicious group manager (i.e., the signer) from falsely accusing the sanitizer to be the source of a signature. Immutability follows from the unforgeability of the regular signature scheme for the fixed parts, and unlinkability from the fact that the sanitizer signs the entire message from scratch (the signature for fixed message parts remains unchanged).

We remark that the actual construction needs a careful implementation of the idea above to make the derived sanitizable signature scheme satisfy all desired security properties. This is in particular true since proposed group signature schemes in the literature like [5|9|22 14 18 19] come with varying security features and set-up assumptions. In this version we thus present a simple but not necessarily the most practical approach to turn our idea into a secure sanitizable scheme, e.g., following the definitions in [3] we do not rely on the fact that public keys of the signer or sanitizer are registered, although this is most likely in practice. In the full version we discuss further variations, e.g., multiple sanitizers, or using a ring signature scheme instead of a group signature scheme, thus dropping the accountability requirement for the derived sanitizable scheme.

Our solution shows that, in general, sanitizable signatures can be built from group signatures, thereby providing a new application for the latter primitive. This relation also immediately gives a feasibility result for sanitizable signatures: Since the work by Bellare et al. [5] about group signatures proves that one can derive them from IND-CCA secure encryption, non-interactive zero-knowledge proofs and digital signatures, all known to exist given trapdoor permutations, it follows that one can also build secure sanitizable signatures from trapdoor permutations.

Organization. In Section 2 we introduce the notion of sanitizable signatures and the security properties given in $[13$. In Section 3 we discuss the notion of unlinkability and its relationship to the other security properties. In Section 4 we present our construction of a secure sanitizable scheme based on group signatures. 


\section{Preliminaries}

In this section we revisit the notion of sanitizable signatures and the previously given security properties.

\subsection{Sanitizable Signatures}

In a sanitizable signature scheme both the signer and the sanitizer hold a key pair $\left(s k_{\text {sig }}, p k_{\text {sig }}\right),\left(s k_{\text {san }}, p k_{\text {san }}\right)$ such that the signer can sign messages with its secret key $s k_{\text {sig }}$ and "attach" a description of the admissible modifications ADM which are allowed to the sanitizer $p k_{\text {san }}$. The sanitizer can then later change such a message according to some modification MOD and update the signature using his secret key $s k_{\text {san }}$. In order to settle disputes about the origin of a messagesignature pair the algorithm Proof enables the signer to produce a proof $\pi$ from previously signed messages that a signature has been created by the sanitizer. This proof can then be verified with the help of the Judge algorithm (but which only needs to decide about the origin in case of a valid message-signature pair in question; for invalid pairs such decisions are in general impossible).

To model admissible modifications we assume that ADM and MOD are (descriptions of) efficient deterministic algorithms such that MOD maps any message $m$ to the modified message $m^{\prime}=\operatorname{MOD}(m)$, and $\operatorname{ADM}(\mathrm{MOD}) \in\{0,1\}$ indicates if the modification is admissible and matches $\mathrm{ADM}$, in which case $\mathrm{ADM}(\mathrm{MOD})=1$. For example, for messages $m=m[1] \ldots m[k]$ divided into blocks $m[i]$ of equal bit length $t$ we can let ADM contain $t$ and the indices of the modifiable blocks, and MOD then essentially consists of pairs $\left(j, m^{\prime}[j]\right)$ defining the new value for the $j$-th block.

For ease of notation we let FIX $_{\mathrm{ADM}}$ be an efficient deterministic algorithm which is uniquely determined by ADM and which maps $m$ to the immutable message part $\operatorname{FIX}_{\mathrm{ADM}}(m)$, e.g., for block-divided messages $\operatorname{FIX}_{\mathrm{ADM}}(m)$ is the concatenation of all blocks not appearing in ADM. To exclude trivial examples we demand that admissible modifications leave the fixed part of a message unchanged, i.e., $\operatorname{FIX}_{\mathrm{ADM}}(m)=\operatorname{FIX}_{\mathrm{ADM}}(\operatorname{MOD}(m))$ for all $m \in\{0,1\}^{*}$ and all MOD with ADM(MOD) = 1. Analogously, to avoid choices like FIX $_{\mathrm{ADM}}$ having empty output, we also require that the fixed part must be "maximal" given $\mathrm{ADM}$, i.e., $\operatorname{FIX}_{\mathrm{ADM}}\left(m^{\prime}\right) \neq \operatorname{FIX}_{\mathrm{ADM}}(m)$ for $m^{\prime} \notin\{\operatorname{MOD}(m) \mid \mathrm{MOD}$ with $\operatorname{ADM}(\mathrm{MOD})=1\}$.

Jumping ahead, we note that for our construction based on group signatures we make another assumption on ADM. This property, denoted modificationdecidability, allows to decide efficiently for given messages $m, m^{*}$ and ADM whether $m^{*}$ is an admissible modification of $m$ with respect to ADM or not. This property is for example satisfied for the block-based approach. However, for our definitions of the security properties and their relationships we do not impose any restriction at this point.

The following definition is taken from [3] :

Definition 1 (Sanitizable Signature Scheme). A sanitizable signature scheme SanSig consists of seven efficient algorithms $\left(K_{\text {Gen }}\right.$ sig,$K_{\text {Gen }}$ san, Sign, Sanit, Verify, Proof, Judge) such that: 
Key Generation. There are two key generation algorithms, one for the signer and one for the sanitizer. Both create a pair of keys, a private key and the corresponding public key:

$$
\left(p k_{\text {sig }}, s k_{\text {sig }}\right) \leftarrow K \operatorname{Ken}_{\text {sig }}\left(1^{n}\right), \quad\left(p k_{\text {san }}, s k_{\text {san }}\right) \leftarrow K \operatorname{Ken}_{\text {san }}\left(1^{n}\right)
$$

Signing. The Sign algorithm takes as input a message $m \in\{0,1\}^{*}$, the secret key sk $k_{\text {sig }}$ of the signer, the public key $p k_{\text {san }}$ of the sanitizer, as well as a description ADM of the admissibly modifiable message parts. It outputs a signature (or $\perp$, indicating an error):

$$
\sigma \leftarrow \operatorname{Sign}\left(m, s k_{s i g}, p k_{\text {san }}, \mathrm{ADM}\right)
$$

We assume that $\mathrm{ADM}$ is recoverable from any signature $\sigma \neq \perp$.

SAnitizing. Algorithm Sanit takes a message $m \in\{0,1\}^{*}$, a signature $\sigma$, the public key $k_{\text {sig }}$ of the signer and the secret key sk $k_{\text {san }}$ of the sanitizer. It modifies the message $m$ according to the modification instruction MOD and determines a new signature $\sigma^{\prime}$ for the modified message $m^{\prime}=\operatorname{MOD}(m)$. Then Sanit outputs $m^{\prime}$ and $\sigma^{\prime}$ (or possibly $\perp$ in case of an error).

$$
\left(m^{\prime}, \sigma^{\prime}\right) \leftarrow \operatorname{Sanit}\left(m, \mathrm{MOD}, \sigma, p k_{\text {sig }}, s k_{\text {san }}\right)
$$

VERIFICATION. The Verify algorithm outputs a bit $d \in\{$ true, false $\}$ verifying the correctness of a signature $\sigma$ for a message $m$ with respect to the public keys $p k_{\text {sig }}$ and $p k_{\text {san }}$.

$$
d \leftarrow \operatorname{Verify}\left(m, \sigma, p k_{\text {sig }}, p k_{\text {san }}\right)
$$

PRoOF. The Proof algorithm takes as input the secret signing key sk $k_{\text {sig }}$, a message $m$ and a signature $\sigma$ as well a set of (polynomially many) additional message-signature pairs $\left(m_{i}, \sigma_{i}\right)_{i=1,2, \ldots, q}$ and the public key $p k_{\text {san }}$. It outputs a string $\pi \in\{0,1\}^{*}$ :

$$
\pi \leftarrow \operatorname{Proof}\left(s k_{\text {sig }}, m, \sigma,\left(m_{1}, \sigma_{1}\right), \ldots,\left(m_{q}, \sigma_{q}\right), p k_{\text {san }}\right)
$$

JUDGE. Algorithm Judge takes as input a message $m$ and a valid signature $\sigma$, the public keys of the parties and a proof $\pi$. It outputs a decision $d \in\{$ Sig, San $\}$ indicating whether the message-signature pair has been created by the signer or the sanitizer:

$$
d \leftarrow \operatorname{Judge}\left(m, \sigma, p k_{\text {sig }}, p k_{\text {san }}, \pi\right)
$$

For a sanitizable signature scheme the usual correctness properties should hold, saying that genuinely signed or sanitized messages are accepted and that a genuinely created proof by the signer leads the judge to decide in favor of the signer. For a formal approach to correctness see [3]. 


\subsection{Security of Sanitizable Signatures}

Here we recall the security notions for sanitizable signatures given by Brzuska et al. [3]. We note that, there, they show that signer and sanitizer accountability together imply unforgeability, and that transparency implies privacy. Hence, in principle it suffices to show immutability, accountability and transparency. We therefore omit the formal definitions of unforgeability and privacy here and refer the reader to the full version of the paper.

Immutability. This property demands informally that a malicious sanitizer cannot change inadmissible blocks. In the attack model below the malicious sanitizer $\mathcal{A}$ interacts with the signer to receive signatures $\sigma_{i}$ for messages $m_{i}$, descriptions $\mathrm{ADM}_{i}$ and keys $p k_{\mathrm{san}, i}$ of its choice, before eventually outputting a valid pair $\left(p k_{\mathrm{san}}^{*}, m^{*}, \sigma^{*}\right)$ such that message $m^{*}$ is not a "legitimate" transformation of one of the $m_{i}$ 's under the same key $p k_{\mathrm{san}}^{*}=p k_{\mathrm{san}, i}$. The latter is formalized by requiring that for each query $p k_{\mathrm{san}}^{*} \neq p k_{\mathrm{san}, i}$ or $m^{*} \notin$ $\left\{\operatorname{MOD}\left(m_{i}\right) \mid \operatorname{MOD}\right.$ with $\left.\mathrm{ADM}_{i}(\mathrm{MOD})=1\right\}$ for the value $\mathrm{ADM}_{i}$ in $\sigma_{i}$, e.g., that for block-divided messages $m^{*}$ and $m_{i}$ differ in at least one inadmissible block. As the adversary can query the signer for several sanitizer keys $p k_{\text {san }}$, the security definition also covers the case where the signer interacts with several sanitizers simultaneously.

Definition 2 (Immutability). A sanitizable signature scheme SanSig is immutable if for any efficient algorithm $\mathcal{A}$ the probability that the following experiment Immutability ${ }_{\mathcal{A}}^{\mathrm{SanSig}}(n)$ returns 1 is negligible (as a function of $n$ ).

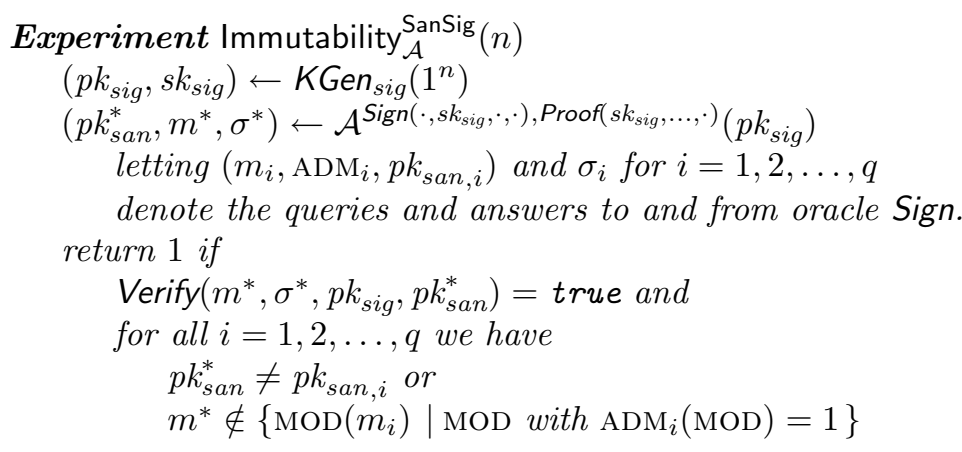

Accountability. Accountability says the origin of a (sanitized) signature should be undeniable. There are the following two types of accountability: sanitizeraccountability says that, if a message has not been signed by the signer, then even a malicious sanitizer should not be able to make the judge accuse the signer. Signer-accountability says that, if a message and its signature have not been sanitized, then even a malicious signer should not be able to make the judge accuse the sanitizer.

In the sanitizer-accountability game let $\mathcal{A}_{\text {Sanit }}$ be an efficient adversary playing the role of the malicious sanitizer. Adversary $\mathcal{A}_{\text {Sanit }}$ has access to a Sign and Proof oracle. Her task is to output a valid message-signature pair $m^{*}, \sigma^{*}$ together with 
a key $p k_{\text {san }}^{*}$ (with $\left(p k_{\text {san }}^{*}, m^{*}\right)$ being different from pairs $\left(m_{i}, p k s a n i\right)$ previously queried to the Sign oracle) such that the proof produced by the signer via Proof still leads the judge to decide "Sig", i.e., that the signature has been created by the signer.

Definition 3 (Sanitizer-Accountability). One calls a sanitizable signature scheme SanSig sanitizer-accountable if for any efficient $\mathcal{A}_{\text {Sanit }}$ the probability

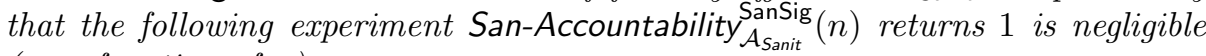
(as a function of $n$ ).

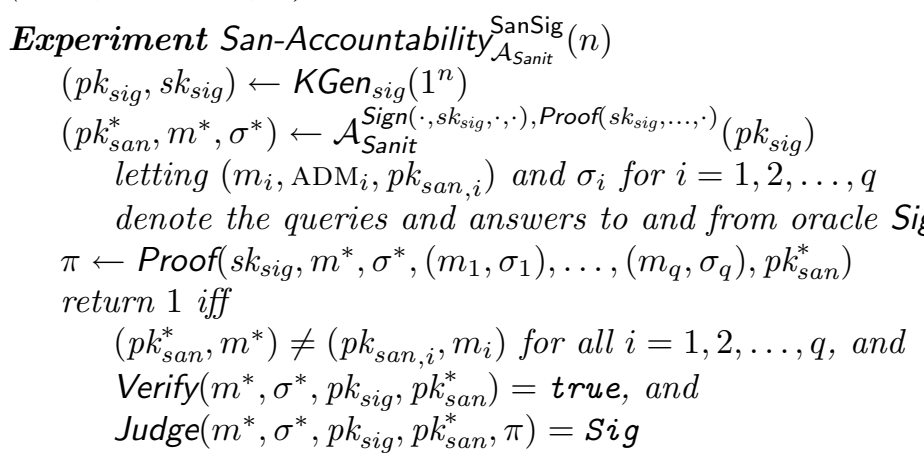

In the signer-accountability game a malicious signer $\mathcal{A}_{\text {sign }}$ gets a public sanitizing key $p k_{\text {san }}$ as input. She is allowed to query a sanitizing oracle about tuples $\left(m_{i}, \operatorname{MOD}_{i}, \sigma_{i}, p k_{\text {sig }_{i}}\right)$ receiving answers $\left(m_{i}^{\prime}, \sigma_{i}^{\prime}\right)$. Adversary $\mathcal{A}_{\text {sign }}$ finally outputs a tuple $\left(p k_{\text {sig }}^{*}, m^{*}, \sigma^{*}\right)$ and is considered to succeed if Judge accuses the sanitizer for the new key-message pair $p k_{\text {sig }}^{*}, m^{*}$ with a valid signature $\sigma^{*}$.

Definition 4 (Signer-Accountability). A sanitizable signature scheme SanSig is called signer-accountable if for any efficient $\mathcal{A}_{\text {sign }}$ the probability that the following experiment Sig-Accountability ${ }_{\mathcal{A}_{\text {sign }}}^{\mathrm{SanSig}}(n)$ returns 1 is negligible (as a function of $n$ ):

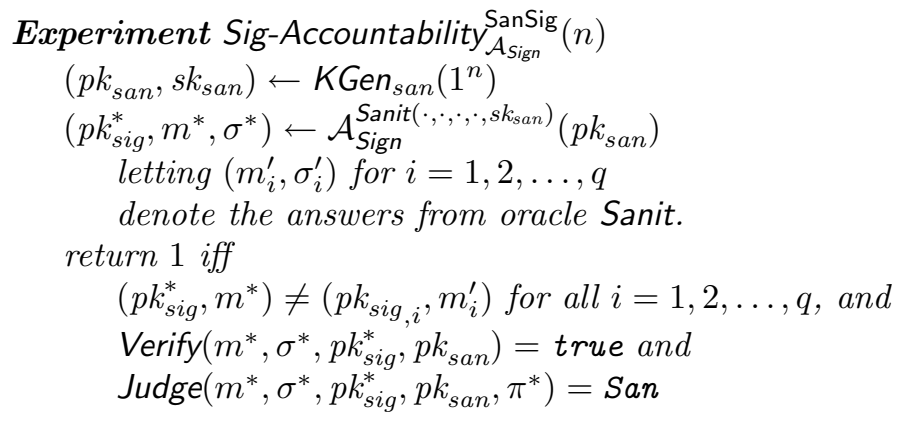

Transparency. We define transparency by the following adversarial game. We consider an adversary $\mathcal{A}$ with access to Sign, Sanit and Proof oracles with which the adversary can create signatures for (sanitized) messages and learn proofs. In addition, $\mathcal{A}$ gets access to a Sanit/Sign box which contains a secret random bit $b \in\{0,1\}$ and which, on input a message $m$, a modification information MOD and a description ADM 
- for $b=0$ runs the signer algorithm to create $\sigma \leftarrow \operatorname{Sign}\left(m, s k_{\mathrm{sig}}, p k_{\mathrm{sig}}, \mathrm{ADM}\right)$, then runs the sanitizer algorithm and returns the sanitized message $m^{\prime}$ with the new signature $\sigma^{\prime}$, and

- for $b=1$ acts as in the case $b=0$ but also signs $m^{\prime}$ from scratch with the signing algorithm to create a signature $\sigma^{\prime}$ and returns the pair $\left(m^{\prime}, \sigma^{\prime}\right)$.

Adversary $\mathcal{A}$ eventually produces an output $a$, the guess for $b$. A sanitizable signature is now said to be transparent if for all efficient algorithms $\mathcal{A}$ the probability for a right guess $a=b$ in the above game is negligibly close to $\frac{1}{2}$. Below we also define a relaxed version called proof-restricted transparency and discuss the idea after the definition.

Definition 5 ((Proof-Restricted) Transparency). A sanitizable signature scheme SanSig is (proof-restricted) transparent if for any efficient algorithm

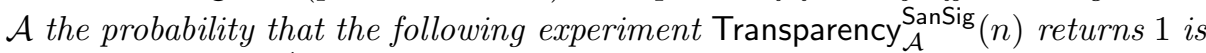
negligibly close to $\frac{1}{2}$.

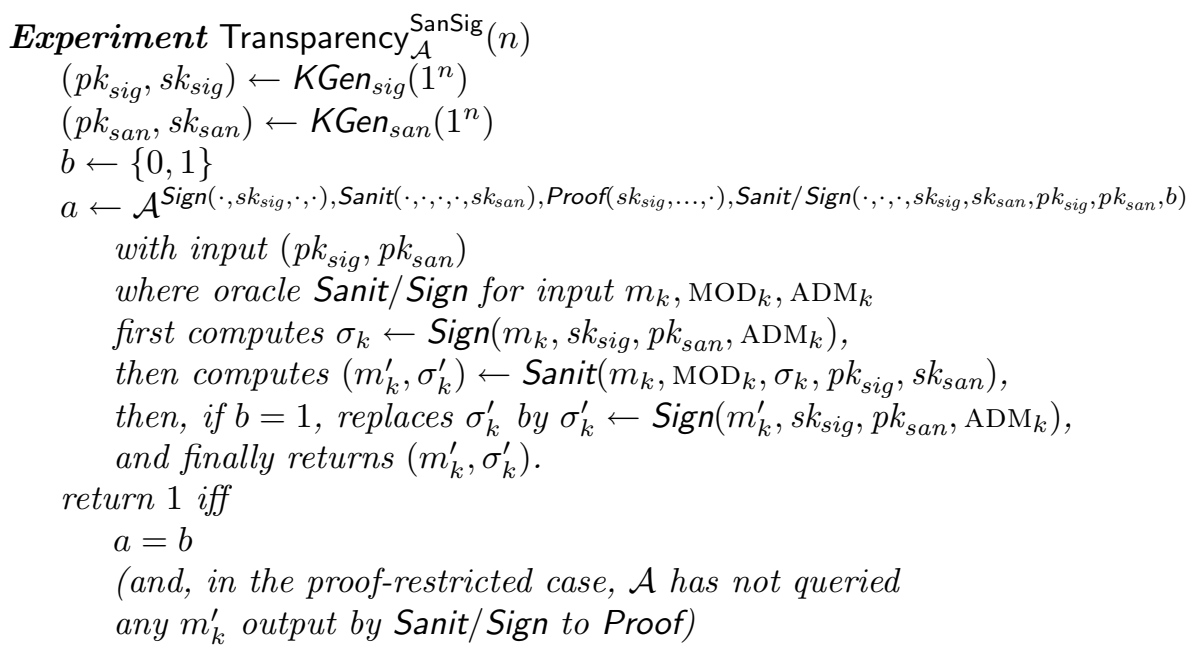

The original definition of Brzuska et al. 3] does not consider the proof-restricted case. Without this restriction, though, achieving transparency at first seems to be impossible because the adversary can then always submit the replies of the Sanit/Sign oracle to the Proof oracle and thereby recover the secret bit $b$. However, in their construction the Proof algorithm searches in the list of previously signed messages and only gives a useful answer if it finds a match, enabling transparency without this restriction. Yet, any solution (like ours here) where the Proof algorithm is "history-free" can only achieve the proof-restricted version. Note that Proof algorithms forgoing the set of previously signed messages are preferable from an efficiency point of view, of course.

As for the implications among the security notions [3] we note that proofrestricted transparency only implies a proof-restricted form of privacy, where the answer messages of the LoRSanit oracle cannot be submitted to the Proof oracle either. However, since we show in the next section that unlinkability implies full 
privacy and our construction achieves unlinkability, our scheme is also private in the non-restricted sense. We note that all the separation results in [3] remain valid for proof-restricted transparency.

\section{Unlinkability}

In this section we define unlinkability formally and discuss its relationship to the other security notions.

\subsection{Definition}

As explained in the introduction, unlinkability refers to the impossibility to use the signatures to identify sanitized message-signature pairs originating from the same source. Technically, we use an indistinguishability-based approach to define this property, saying that, given a signature for a sanitized message of two possible sources, the adversary cannot predict the actual original message better than by guessing. This should even hold if the adversary herself provides the two source message-signature pairs and modifications of which one is sanitized. The stipulation here is that the two modifications yield the same sanitized message. Else, if for example the sanitized messages still contain some unique but distinct entry, then predicting the source is easy, of course. This, however, is beyond the scope of signature schemes: the scheme should only prevent that signatures can be used to link data.

Formally, we use a game-based approach to define unlinkability, similar to the other security notions in [3. The adversary is given access to a signing oracle and a sanitizer oracle (and a proof oracle since this step depends on the signer's secret key and may leak valuable information). The adversary is also allowed to query a left-or-right oracle LoRSanit which is initialized with a secret random bit $b$. In each of the multiple queries to LoRSanit the adversary provides a pair of tuples, each consisting of a message, a modification and a valid signature, such that the recoverable description of admissible modifications is identical in both cases (since we assume that ADM is recoverable from a signature providing distinct descriptions ADM would allow a trivial attack; so would the case that only one signature is valid). Depending on the bit $b$, the adversary gets the sanitized message-signature pair of either the left or right input pair. The adversary should eventually predict the bit $b$ significantly better than with the guessing probability of $\frac{1}{2}$.

Definition 6 (Unlinkability). A sanitizable signature scheme SanSig is unlinkable if for any efficient algorithm $\mathcal{A}$ the probability that the following experiment Unlinkability $\mathcal{S}_{\mathcal{A}}^{\mathrm{SanSig}}(n)$ returns 1 is negligibly close to $\frac{1}{2}$.

$$
\begin{aligned}
& \text { Experiment Unlinkability } \operatorname{SanSig}_{\mathcal{A}}(n) \\
& \left(p k_{\text {sig }}, s k_{\text {sig }}\right) \leftarrow K \mathrm{KGn}_{\text {sig }}\left(1^{n}\right) \\
& \left(p k_{\text {san }}, s k_{\text {san }}\right) \leftarrow K \mathrm{KGen}_{\text {san }}\left(1^{n}\right) \\
& b \leftarrow\{0,1\}
\end{aligned}
$$




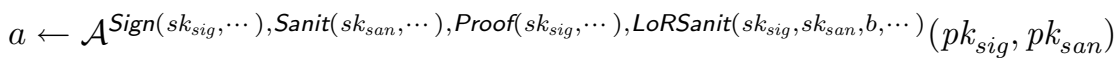

where oracle LoRSanit $\left(\cdot, \cdot, \cdot, s k_{\text {sig }}, k_{\text {san }}, b\right)$, on input

$\left(m_{j, 0}, \mathrm{MOD}_{j, 0}, \sigma_{j, 0}, m_{j, 1}, \mathrm{MOD}_{j, 1}, \sigma_{j, 1}\right)$ with recoverable $\mathrm{ADM}_{j, 0}=\mathrm{ADM}_{j, 1}$

$\operatorname{Verify}\left(m_{j_{0}}, \sigma_{j, 0}, p k_{\text {sig }}, p k_{\text {san }}\right)=\operatorname{true}, \operatorname{Verify}\left(m_{j_{1}}, \sigma_{j, 1}, p k_{\text {sig }}, p k_{\text {san }}\right)=$ true, returns $\left(m_{j}^{\prime}, \sigma_{j}^{\prime}\right) \leftarrow \operatorname{Sanit}\left(m_{j, b}, \operatorname{MOD}_{j, b}, \sigma_{j, b}, p k_{s i g}, s k_{s a n}\right)$,

and where $\left(m_{j, 0}, \mathrm{MOD}_{j, 0}, \mathrm{ADM}_{j, 0}\right) \equiv\left(m_{j, 1}, \mathrm{MOD}_{j, 1}, \mathrm{ADM}_{j, 1}\right)$,

i.e., are mapped to the same modified message.

return 1 if $a=b$.

A pictorial description is given in Figure 2. We note that the definition above is for example robust concerning several sanitization steps in the LoRSanit oracle. That is, we could allow the adversary in the experiment above to submit arbitrarily long "modification chains" $\operatorname{MOD}_{j, 0}^{1}, \ldots, \mathrm{MOD}_{j, 0}^{m}$ and $\mathrm{MOD}_{j, 1}^{1}, \ldots, \mathrm{MOD}_{j, 1}^{m}$ such that the two source documents are gradually sanitized with a match in the resulting documents. Still, predicting $b$ remains hard, as such chains can potentially be simulated by calling the sanitizer oracle for the first $m-1$ modifications manually, before entering the final sanitization step into the LoRSanit oracle.

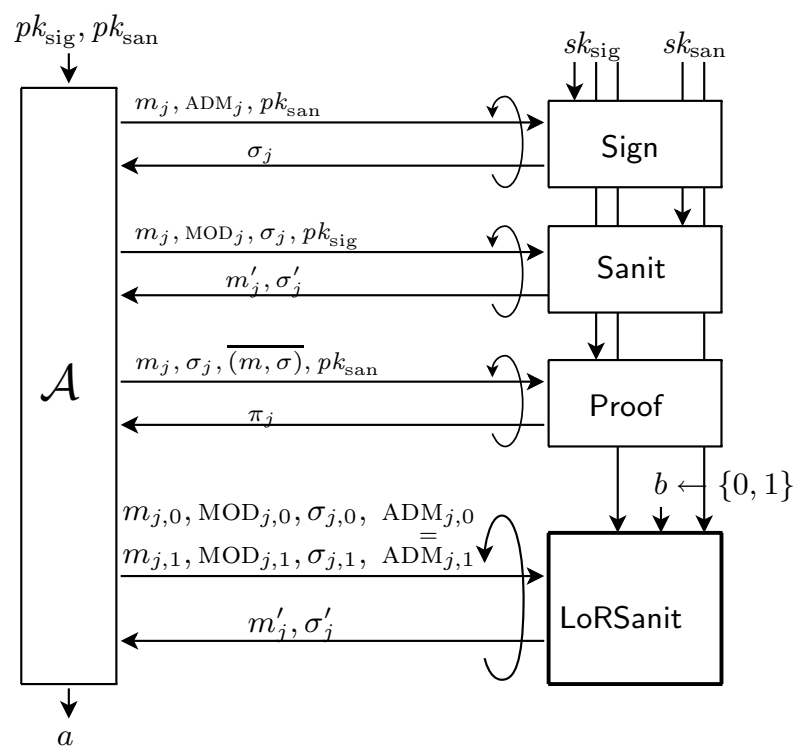

Fig. 2. Unlinkability. $\mathcal{A}$ wins if it outputs $a=b$.

Recall the example of medical records which are sanitized twice, one time basically removing the personal information and the other time removing the medical data. Our notion of unlinkability can then be used to show that such sanitized message-signature pairs do not allow to reconstruct the full data better than by guessing. Assume for simplicity that we only have two records with entries (name\#0, data\#0) and (name\#1, data\#1). Then we create all four possible 
combinations (name\#a, data\#b) for $a, b \in\{0,1\}$ and ask for signatures for them (with both parts being admissibly changeable). For each $a \in\{0,1\}$ we then insert the pairs (name\#a, data\#0) and (name\#a, data\#1) twice into the LoRSanit oracle, one time cutting off the name-part, the other time removing the data-part. Altogether we make thus four calls to the LoRSanit oracle, and we hand those four replies to the adversary. Our unlinkability definition says that one cannot distinguish the two cases (left or right sanitization) better than by guessing, thus also disallowing to tell which data belong to whose name.

Our definition above is for unlinkability with respect to message-signature pairs sanitized by the same sanitizer. One can easily extend the above definition by demanding that the adversary can also determine different sanitizers for the left and for the right input data. But then both sanitizers must have been declared to have the permission to sanitize, otherwise one could easily determine the secret bit of the LoRSanit by picking an invalid sanitizer for one of the input tuples.

\subsection{Relationships of the Security Notions}

We first show that unlinkability does not follow from any of the other security requirements. Then we prove that unlinkability implies privacy, and finally discuss that unlinkability does not imply any of the other properties.

Proposition 1 (Independence of Unlinkability). Assume that there exists a sanitizable signature scheme (obeying one or more of the properties unforgeability, immutability, privacy, (proof-restricted) transparency, signer-accountability and sanitizer-accountability). Then there exists a sanitizable signature scheme which is not unlinkable but preserves the other security properties.

The proof follows by simply appending a unique identifier id to each signature. This does not destroy any of the other security properties but clearly violates unlinkability. The proof of the following is straightforward as the privacy experiment is essentially the unlinkability experiment with less control for the adversary:

Proposition 2 (Unlinkability Implies Privacy). Any unlinkable sanitizable signature scheme is also private.

With the next proposition we show that unlinkability does not imply any of the other security properties (assuming that we start with a secure sanitizable signature scheme like the one we construct in the next section):

Proposition 3 (Independence of Other Properties). Assume that there exists a sanitizable signature scheme which is unforgeable, immutable, private, (proof-restricted) transparent, signer-accountable, sanitizer-accountable and unlinkable. Then for any of the properties immutability, transparency, unlinkability, signer-accountability and sanitizer-accountability, there exists a sanitizable signature scheme obeying all properties except for the one in question. 
Proof. The fact that unlinkability does not follow from the other properties has already been shown in Proposition 1. For the other properties we remark that the counterexamples in [3] which seperate immutability, transparency, signeraccountability and sanitizer-accountability from the other properties also preserve unlinkability in each case (and also hold for proof-restricted transparency).

\section{Constructions Based on Group Signatures}

In this section we present our unlinkable sanitizable signature scheme (which also satisfies the other security properties). As explained in the introduction, the idea is to use a group signature scheme for the group consisting of the signer and the sanitizer, such that the signer signs the immutable message part with a regular signature scheme and the full message with the group signature scheme. The sanitizer can then update the full message and only sign this second component. The signer also takes over the role of the group manager in order to provide accountability.

\subsection{Group Signatures}

Group signatures, introduced by Chaum and van Heyst [13, allow a set of users to sign on behalf of the group such that outsiders cannot distinguish between different signers (anonymity) but such that a group manager can trace the signer's identity (traceability). We follow the formal framework of Bellare et al. [5] but add the non-frameability requirement [9] that even the group manager cannot sign on behalf of the users. Recall that this is necessary for the accountability in our sanitizable signature scheme, where the signer acts as the group manager and should not be able to make the judge falsely accuse the sanitizer.

We briefly recall group signature schemes and their security properties. For comprehensive definitions see the full version of the paper and [5]. A group signature scheme GS consists of six efficient algorithms GS = (GKGen, UKGen, GSig, GVf, Open, GJudge) where

- $\left(s k_{\text {user }}, p k_{\text {user }}\right) \leftarrow U K G e n\left(1^{n}\right)$ generates individual user key pairs,

- $(g m s k, g p k$, cert $) \leftarrow$ GKGen $\left(1^{n}, \mathbf{g p k}_{u s e r}\right)$ takes the tuple $\mathbf{g p k}_{\text {user }}$ of the users' public keys and generates a group manager secret key gmsk, a group public key $g p k$ and an individual certificate cert $_{i}$ for each user, where cert designates the tuple of all cert $_{i}$,

- $\sigma \leftarrow \operatorname{GSig}\left(s k_{\mathrm{user}, i}, \operatorname{cert}_{i}, g p k, m\right)$ signs a message $m$ given the user's secret data $s k_{\text {user }, i}$, cert $_{i}$ and the group's public key $g p k$,

$-(i, \pi) \leftarrow$ Open $\left(g m s k, m, \sigma, \mathbf{g p k}_{u s e r}, g p k\right)$ on input a message $m$ and signature $\sigma$ returns the index $i$ of the alleged signer and a proof $\pi$ such that

- GJudge $\left(m, \sigma, i, \pi, g p k, \mathbf{g p k}_{u s e r}\right)$ either confirms the accusation or denies it.

There are three security properties for group signatures $[5] 9$ : 
Anonymity. Means that one cannot tell from a group signature who signed a message, even if one knows the secret data of the user and can ask the group manager to reveal the identities for other signatures.

TraceABILITY. Refers to the fact that a malicious user cannot falsely accuse an honest user to be the signer of a message, even if the malicious user is allowed to see other signatures generated by this honest user and can call the group manager.

Non-Frameability. Strengthens traceability in the sense that even if the malicious user colludes with the group manager they cannot frame an honest user.

Definition 7 (Secure Group Signature). We call a group signature scheme secure if it is anonymous and non-frameable.

Note that we tailor the group signature definitions to our needs thereby adding non-frameability, making the scheme syntax setup session free and relaxing the security model concerning some technical issues which are discussed in the full version of this paper. As for instantiations we remark that the (generic) construction by Bellare et al. [5] satisfies our adapted definitions. As for more efficient group signature schemes, we can implement our sanitizable signature scheme with other group signature schemes like 22 141819. Yet, these group signature schemes need additional set-up assumptions like a trusted party generating common parameters or interactive registration of users. Our sanitizable signature scheme then inherits these characteristics (recall that, in practice, registration of signer and sanitizer keys is for example necessary to provide meaningful accountability).

\subsection{Construction}

In this section we show that the new security requirement of unlinkability can be achieved in combination with the five established security properties formalized in [3. Recall that we sign the entire message, including the modifiable parts, with the group signature scheme, and - in order to prevent inadmissible changesthe signer also signs the fixed part with a regular scheme. This requires some care because if we take an arbitrary signature scheme then the signature itself may act as a unique identifier, even for messages with identical fixed parts. Thereby, unlinkability would be violated.

The solution is to use a secure deterministic signature scheme for the fixed part (such that the signature is identical for messages with the same fixed part). Alternatively, one can deploy a rerandomizable signature scheme such that the sanitizer can rerandomize the signature, excising the link to the input signature. Below we use the "deterministic solution" for simplicity, and since every secure signature scheme can be easily turned into a deterministic one via pseudorandom functions [15].

For a formal definition of strongly unforgeable signature schemes see [17. We need this unforgeability notion (saying that one cannot even find a new signature for a previously signed message) to provide unlinkability. Examples of 
signature schemes achieving this strong notion are $6[12 / 428]$. Moreover, it is possible to obtain a strongly unforgeable signature scheme out of any unforgeable signature scheme applying the transformation of Bellare and Shoup [7. Applying the transformation of [15] one can then make such schemes also deterministic.

Recall that the idea behind our scheme is that for each signature the signer uses a group manager key, creates a certified user key to sign the modifiable parts, and certifies the sanitizer's public key as a group member to support modifications. But since our definition of sanitizable signatures demands statefree solutions, the signer formally cannot store the group manager key for this sanitizer and would need to create a new one for each call. We bypass this as follows: we let the signer for each signing request, including a public key of the sanitizer $p k_{\text {san }}$, create the group manager's keys etc. via the corresponding group signature algorithms, but provide the randomness for these algorithms by applying a pseudorandom function to $p k_{\text {san }}$ (see [16] for a definition of pseudorandom functions). By this, we end up with (almost) independent keys for different sanitizers, but use consistent parameters for each sanitizer. For the same reason we also include the group membership certificate of the sanitizer in the signature, although it would be given directly to the sanitizer instead. As a side effect, since the group manager's public key is tied to the sanitizer in question, we also rely on group signatures with static joins only.

Construction 1 (Sanitizable Signature Scheme). Let $\mathcal{S}=($ SKGen, SSign, SVf) be a (regular) signature scheme, let GS = (GKGen, UKGen, GSig, GVf, Open, GJudge) be a group signature scheme. Let $\mathcal{P} \mathcal{R} \mathcal{F}=\left(K_{\text {Gen }}\right.$ prf, PRF $)$ be pseudorandom function. Define the sanitizable signature scheme SanSig = (KGen $_{\text {sig }}, K_{\text {Gen }}$ san, Sign, Sanit, Verify, Proof, Judge) as follows:

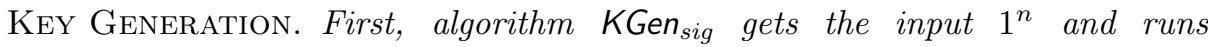
$(s s k, s p k) \leftarrow \operatorname{SKGen}\left(1^{n}\right)$ to create a key pair for the signature scheme, and then also invokes $k \leftarrow K G e n_{\text {prf }}\left(1^{n}\right)$ to derive a key for the pseudorandom function. It outputs $\left(s k_{\text {sig }}, p k_{\text {sig }}\right)=((s s k, k), s p k)$. Algorithm KGen $\operatorname{Kan}_{\text {san }}\left(1^{n}\right)$ generates a key pair $\left(s k_{\text {san }}, p k_{\text {san }}\right)=\left(g s k_{\text {san }}, g p k_{\text {san }}\right) \leftarrow U K G e n\left(1^{n}\right)$ of the group signature scheme.

Signing. Algorithm Sign on input $m \in\{0,1\}^{*}, s k_{\text {sig }}=(s s k, k), p k_{\text {san }}$, ADM sets $m_{\mathrm{FIX}}=\operatorname{FIX}_{\mathrm{ADM}}(m)$ for the algorithm $\mathrm{FIX}_{\mathrm{ADM}}$ determined by $\mathrm{ADM}$. It runs the user key generation algorithm $\left(g_{s k_{\text {sig }}}, g p k_{\text {sig }}\right) \leftarrow U K G e n\left(1^{n} ; \operatorname{PRF}\left(k, 0 \| p k_{\text {san }}\right)\right)$ for randomness $\operatorname{PRF}\left(k, 0 \| p k_{\text {san }}\right)$ and afterwards the group manager algorithm to compute

$$
\left(\text { gmsk }_{\text {gpk }}, \operatorname{cert}_{\text {sig }}, \operatorname{cert}_{\text {san }}\right) \leftarrow G K G e n\left(1^{n},\left(g p k_{\text {sig }}, p k_{\text {san }}\right) ; \operatorname{PRF}\left(k, 1 \| p k_{\text {san }}\right)\right)
$$

for randomness $\operatorname{PRF}\left(k, 1 \| p k_{\text {san }}\right)$. It computes

$$
\begin{gathered}
\sigma_{\mathrm{FIX}}=\operatorname{SSign}\left(s s k,\left(m_{\mathrm{FIX}}, \mathrm{ADM}, p k_{\text {san }}, g p k\right)\right) \text { and } \\
\sigma_{\mathrm{FULL}}=G \operatorname{Sig}\left(g s k_{\text {sig }}, \operatorname{cert}_{\text {sig }},\left(m, p k_{\text {sig }}\right), g p k\right)
\end{gathered}
$$

using the signing algorithms of the regular and of the group signature scheme. The algorithm finally returns $\sigma=\left(\sigma_{\mathrm{FIX}}, \sigma_{\mathrm{FULL}}, \mathrm{ADM}, p k_{\text {san }}\right.$, cert $_{\text {san }}$, gpk $)$. 
SAnitizing. Algorithm Sanit on input a message $m$, information MOD, a signature $\sigma=\left(\sigma_{\mathrm{FIX}}, \sigma_{\mathrm{FULL}}, \mathrm{ADM}, p k_{\text {san }}\right.$, cert $\left._{\text {san }}, g p k\right)$, keys $p k_{\text {sig }}$ and $s k_{\text {san }}$ first recovers $m_{\mathrm{FIX}}=\operatorname{FIX}_{\mathrm{ADM}}(m)$. It then checks that $\mathrm{MOD}$ is admissible according to $\mathrm{ADM}$ and that $\sigma_{\mathrm{FIX}}$ is a valid signature for message ( $\left.m_{\mathrm{FIX}}, \mathrm{ADM}, p k_{\text {san }}, g p k\right)$ under key spk. If not, it stops outputting $\perp$. Else, it derives the modified message $m^{\prime}=\operatorname{MOD}(m)$ and computes

$$
\sigma_{\mathrm{FULL}}^{\prime}=\operatorname{GSig}\left(g s k_{\text {san }}, \operatorname{cert}_{\text {san }},\left(m^{\prime}, p k_{\text {sig }}\right), g p k\right)
$$

and outputs $m^{\prime}$ together with $\sigma^{\prime}=\left(\sigma_{\mathrm{FIX}}, \sigma_{\mathrm{FULL}}^{\prime}, \mathrm{ADM}, p k_{\text {san }}\right.$, cert $\left._{\text {san }}, g p k\right)$.

VerificAtion. Algorithm Verify gets as input a message $m \in\{0,1\}^{*}$, a signature $\sigma=\left(\sigma_{\mathrm{FIX}}, \sigma_{\mathrm{FULL}}, \mathrm{ADM}, p k_{\text {san }}\right.$, cert $_{\text {san }}$, gpk $)$ and public keys $p k_{\text {sig }}=$ spk and $p k_{\text {san }}$. It first recovers $m_{\mathrm{FIX}}=\operatorname{FIX}_{\mathrm{ADM}}(m)$. It then checks whether SVf $($ spk, $\left.\left(m_{\mathrm{FIX}}, \mathrm{ADM}, p k_{\text {san }}, g p k\right), \sigma_{\mathrm{FIX}}\right)=1$ and $\mathrm{GVf}\left(g p k,\left(m, p k_{\text {sig }}\right), \sigma_{\mathrm{FULL}}\right)$ verifies under the group public key as true, too. If so, it outputs 1 , declaring the entire signature as valid. Otherwise it returns 0 , indicating an invalid signature.

PROOF. Algorithm Proof gets as input sk $k_{\text {sig }}, m$ and $\sigma=\left(\sigma_{\mathrm{FIX}}, \sigma_{\mathrm{FULL}}, \mathrm{ADM}, p k_{\text {san }}\right.$, cert $\left._{\text {san }}, g p k\right)$. It parses the key as $s k_{\text {sig }}=(s s k, k)$ and recomputes

$$
\left(g_{m s k}, g p k^{\prime}, \operatorname{cert}_{s i g}^{\prime}, \operatorname{cert}_{s a n}^{\prime}\right)=G K G e n\left(1^{n},\left(g p k_{s i g}, p k_{s a n}\right) ; \operatorname{PRF}\left(k, 1 \| p k_{\text {san }}\right)\right)
$$

and checks that gpk' $k^{\prime}$ gpk and cert $_{\text {san }}^{\prime}=$ cert $_{\text {san }}$ (and immediately returns $\perp$ if not $)$. It next verifies that $S V f\left(s p k,\left(m_{\mathrm{FIX}}, \mathrm{ADM}, p k_{\text {san }}, g p k\right), \sigma_{\mathrm{FIX}}\right)=1$ and, if so, computes and outputs $(i, \pi) \leftarrow$ Open $\left(g m s k,\left(m, p k_{\text {sig }}\right), \sigma_{\mathrm{FULL}}, g p k\right)$, where $i \in\{$ Sig, San $\}$ is the identity returned by the Open algorithm (or, Proof returns $\perp$ if any of the verification steps above fail).

JUDGE. The judge on input $m, \sigma, p k_{\text {sig }}, p k_{\text {san }}$ and a proof $(i, \pi)$ with $i \in$ $\left\{\right.$ Sig, San\} parses $\sigma$ as $\left(\sigma_{\mathrm{FIX}}, \sigma_{\mathrm{FULL}}, \mathrm{ADM}, p k_{\text {san }}\right.$, cert $\left._{\text {san }}, g p k\right)$. It derives $b \leftarrow$ GJudge $\left(\left(m, p k_{\text {sig }}\right), \sigma_{\mathrm{FULL}}, i, \pi, g p k\right)$ using the judge algorithm of the group signature scheme. If $b=$ true it outputs $i$, else it outputs $i=S i g$.

Completeness of signatures generated by the signer and sanitizer follows easily from the completeness of the underlying signature schemes and the fact that FIX $_{\mathrm{ADM}}$ leaves the fixed message parts unchanged for modified messages. There is a negligible probability that a signature of the signer or the sanitizer also verifies under the other party's other key, yielding possibly a wrong answer from the judge. We ignore this issue here for simplicity.

\subsection{Security Proof}

We need an additional property of the admissible modifications ADM: given arbitrary messages $m, m^{*} \in\{0,1\}^{*}$ (and a security parameter $1^{n}$ ) it should be efficiently decidable whether $m^{*} \in\{\operatorname{MOD}(m) \mid \operatorname{MOD}$ with $\operatorname{ADM}(\operatorname{MOD})=1\}$ or not. We call such ADM modification-decidable and a sanitizable signature scheme modification-restricted if it only allows modification-decidable ADM. As an example consider again block-divided messages where ADM describes the block-length and the indices of changeable blocks. Then it is easy to check whether $m^{*}$ has been changed in admissible blocks only or not. 
Theorem 2. Let $\mathcal{S}$ be a strongly unforgeable deterministic signature scheme and let GS be a secure group signature scheme. Assume further that $\mathcal{P} \mathcal{R} \mathcal{F}$ is a pseudorandom function. Then the modification-restricted sanitizable signature scheme in Construction 1 is unforgeable, immutable, private, proof-restricted transparent, accountable and unlinkable.

As unlinkability implies privacy, and as moreover, sanitizer-accountability and signer-accountability imply unforgeability, it suffices to prove these two types of accountability as well as with unlinkability, immutability and (proof-restricted) transparency.

For the proof idea note that we can reduce transparency of our sanitizable signatures to anonymity of the underlying group signature scheme. Traceability of the group signature scheme enables the group manager (i.e., the signer) to provide a proof that a message has indeed been signed by a certain group member. Thus, if the sanitizer signs a message, the signer can produce evidence that this signature originates from the sanitizer. This shows sanitizer-accountability. Vice versa, the unframeability property of group signature scheme assures that the group manager (i.e., the signer) cannot falsely accuse a group member of having signed a message. Therefore, signer-accountability follows from unframeability.

The unforgeability of the underlying regular signature scheme assures immutability: If the sanitizer changed admissible parts of a message, she would be obliged to forge a signature for the fixed part. Unlinkability holds as the sanitizer creates a new group signature from scratch when sanitizing. Furthermore, the signature of the regular signature scheme remains unchanged, and is identical for different documents with the same fixed part because we use a deterministic scheme. The formal proof follows these ideas and appears in the full paper.

\section{Acknowledgments}

We thank the anonymous reviewers for valuable comments. Marc Fischlin, Anja Lehmann and Dominique Schröder are supported by the Emmy Noether Program Fi 940/2-1 of the German Research Foundation (DFG). This work was also supported by CASED (www. cased.de).

\section{References}

1. Ateniese, G., Chou, D.H., de Medeiros, B., Tsudik, G.: Sanitizable Signatures. In: di Vimercati, S.D.C., Syverson, P.F., Gollmann, D. (eds.) ESORICS 2005. LNCS, vol. 3679, pp. 159-177. Springer, Heidelberg (2005)

2. Boneh, D., Boyen, X.: Short Signatures Without Random Oracles. In: Cachin, C., Camenisch, J.L. (eds.) EUROCRYPT 2004. LNCS, vol. 3027, pp. 56-73. Springer, Heidelberg (2004)

3. Brzuska, C., Fischlin, M., Freudenreich, T., Lehmann, A., Page, M., Schelbert, J., Schroeder, D., Volk, F.: Security of Sanitizable Signatures Revisited. In: Jarecki, S., Tsudik, G. (eds.) PKC 2009. LNCS, vol. 5443, pp. 317-336. Springer, Heidelberg (2009) 
4. Boneh, D., Lynn, B., Shacham, H.: Short Signatures from the Weil Pairing. Journal of Cryptology 17(4), 297-319 (2004)

5. Bellare, M., Micciancio, D., Warinschi, B.: Foundations of Group Signatures: Formal Definitions, Simplified Requirements, and a Construction Based on General Assumptions. In: Biham, E. (ed.) EUROCRYPT 2003. LNCS, vol. 2656, pp. 614-629. Springer, Heidelberg (2003)

6. Bellare, M., Rogaway, P.: The Exact Security of Digital Signatures - How to Sign with RSA and Rabin. In: Maurer, U.M. (ed.) EUROCRYPT 1996. LNCS, vol. 1070, pp. 399-416. Springer, Heidelberg (1996)

7. Bellare, M., Shoup, S.: Two-Tier Signatures, Strongly Unforgeable Signatures, and Fiat-Shamir Without Random Oracles. In: Okamoto, T., Wang, X. (eds.) PKC 2007. LNCS, vol. 4450, pp. 201-216. Springer, Heidelberg (2007)

8. Boneh, D., Shen, E., Waters, B.: Strongly Unforgeable Signatures Based on Computational Diffie-Hellman. In: Yung, M., Dodis, Y., Kiayias, A., Malkin, T.G. (eds.) PKC 2006. LNCS, vol. 3958, pp. 229-240. Springer, Heidelberg (2006)

9. Bellare, M., Shi, H., Zhang, C.: Foundations of Group Signatures: The Case of Dynamic Groups. In: Menezes, A. (ed.) CT-RSA 2005. LNCS, vol. 3376, pp. 136-153. Springer, Heidelberg (2005)

10. Canard, S., Jambert, A.: On Extended Sanitizable Signature Schemes. In: Pieprzyk, J. (ed.) CT-RSA 2010. LNCS, vol. 5985, pp. 179-194. Springer, Heidelberg (2010)

11. Canard, S., Laguillaumie, F., Milhau, M.: Trapdoor Sanitizable Signatures and Their Application to Content Protection. In: Bellovin, S.M., Gennaro, R., Keromytis, A.D., Yung, M. (eds.) ACNS 2008. LNCS, vol. 5037, pp. 258-276. Springer, Heidelberg (2008)

12. Coron, J.-S.: On the Exact Security of Full Domain Hash. In: Bellare, M. (ed.) CRYPTO 2000. LNCS, vol. 1880, pp. 229-235. Springer, Heidelberg (2000)

13. Chaum, D., van Heyst, E.: Group Signatures. In: Davies, D.W. (ed.) EUROCRYPT 1991. LNCS, vol. 547, pp. 241-246. Springer, Heidelberg (1991)

14. Delerablée, C., Pointcheval, D.: Dynamic Fully Anonymous Short Group Signatures. In: Nguyên, P.Q. (ed.) VIETCRYPT 2006. LNCS, vol. 4341, pp. 193-210. Springer, Heidelberg (2006)

15. Goldreich, O.: Two Remarks Concerning the Goldwasser-Micali-Rivest Signature Scheme. In: Odlyzko, A.M. (ed.) CRYPTO 1986. LNCS, vol. 263, pp. 104-110. Springer, Heidelberg (1987)

16. Goldreich, O.: The Foundations of Cryptography, vol. 1. Cambridge University Press, Cambridge (2001)

17. Goldreich, O.: The Foundations of Cryptography, vol. 2. Cambridge University Press, Cambridge (2004)

18. Groth, J.: Simulation-Sound NIZK Proofs for a Practical Language and Constant Size Group Signatures. In: Lai, X., Chen, K. (eds.) ASIACRYPT 2006. LNCS, vol. 4284, pp. 444-459. Springer, Heidelberg (2006)

19. Groth, J.: Fully Anonymous Group Signatures Without Random Oracles. In: Kurosawa, K. (ed.) ASIACRYPT 2007. LNCS, vol. 4833, pp. 164-180. Springer, Heidelberg (2007)

20. Johnson, R., Molnar, D., Song, D.X., Wagner, D.: Homomorphic Signature Schemes. In: Preneel, B. (ed.) CT-RSA 2002. LNCS, vol. 2271, pp. 244-262. Springer, Heidelberg (2002)

21. Klonowski, M., Lauks, A.: Extended Sanitizable Signatures. In: Rhee, M.S., Lee, B. (eds.) ICISC 2006. LNCS, vol. 4296, pp. 343-355. Springer, Heidelberg (2006) 
22. Kiayias, A., Yung, M.: Group Signatures with Efficient Concurrent Join. In: Cramer, R. (ed.) EUROCRYPT 2005. LNCS, vol. 3494, pp. 198-214. Springer, Heidelberg (2005)

23. Miyazaki, K., Susaki, S., Iwamura, M., Matsumoto, T., Sasaki, R., Yoshiura, H.: Digital documents sanitizing problem. Technical Report ISEC2003-20. IEICE (2003)

24. Steinfeld, R., Bull, L., Zheng, Y.: Content Extraction Signatures. In: Kim, K.-c. (ed.) ICISC 2001. LNCS, vol. 2288, pp. 285-304. Springer, Heidelberg (2002) 